not predictive of seizure outcome included infantile onset and family history of epilepsy. (Spooner CG, Berkovic SF, Mitchell LA, Wrennall JA, Harvey AS. New-onset temporal lobe epilepsy in children. Lesion on MRI predicts poor seizure outcome. Neurology Dec (2 of 2) 2006;67:2147-2153). (Reprints: Dr AS Harvey, Children's Epilepsy Program, Department of Neurology, Royal Children's Hospital (Melbourne), Flemington Road, Parkville, 3052, Australia).

COMMENT. Spontaneous remission of seizures occurs in one-third of patients with new-onset TLE. A lesion on MRI and focal slowing on EEG are predictive of refractory seizures and the need for surgical resection. Whereas $67 \%$ of TLE patients having MRI lesions became seizure free after surgical resection, only $53 \%$ of patients with negative neuroimaging became seizure free with medication alone, at 10 or more year follow-up. (Mathern GW, Trevathan E. Editorial. Neurology Dec 2006;67:2117-2118). Repeat neuroimaging may uncover developing lesions in $14 \%$ of patients with TLE, and prompt surgical evaluation and treatment.

\title{
ELECTROCLINICAL MANIFESTATIONS OF TEMPORAL LOBE EPILEPSY
}

The relationships between etiology, age at onset and electroclinical findings were examined in 77 children with temporal lobe epilepsy (TLE) reported from University of Verona, Verona, and Niguarda Hospital, Milan, Italy. Age at onset was < 3 years in 39 patients, 3 to 6 years in 17, and $>6$ years in 21 . Seizures began with staring, lip cyanosis, and oral automatisms; auras were more common after age 6 years. Video-EEG recordings documented seizures starting independently in both temporal lobes. Three subgroups of TLE were recognized: 1) Symptomatic TLE caused by cortical malformation or tumor; 2) TLE with mesial temporal sclerosis; and 3) cryptogenic TLE. Thirty two patients underwent surgical treatment. (Fontana E, Negrini F, Francione S et al. Temporal lobe epilepsy in children: Electroclinical study of 77 cases. Epilepsia Dec 2006;47:26-30). (Reprints: Dr Elena Fontana, Servizio di Neuropsichiatria infantile, Policlinico GB Rossi, P le LA, Scuro, 108-37134 Verona, Italy).

COMMENT. Video-EEG recording of seizures is essential for correct diagnosis and localization of TLE in young children. In cases lacking neuroradiological correlation, a repeat video-EEG may be needed at follow-up. Wyllie E et al (Epilepsia 1993;34:859-868), in a previous electroclinical study of 14 children aged 16 months to 12 years, also demonstrated the value of video-EEG recordings in pre-surgical localization of the epileptogenic zone in children with TLE, especially in cases with mesial sclerosis and without tumor. In 9 with low grade neoplasms, the EEG findings were complex, including multifocal interictal sharp waves or falsely lateralized EEG seizure onset. An excellent surgical outcome is reported in 19 patients with mesial temporal sclerosis following prolonged complex febrile seizures and treated at the Montreal Neurological Institute (AbouKhalil B, Andermann F et al. Epilepsia 1993;34:878). Ictal SPECT was superior to ictal EEG in localizing value in a study of 15 children with TLE, aged 7-14 years, at Royal Children's Hospital, Melbourne, Australia (Harvey AS et al. Epilepsia 1993;34:869). 